\title{
Noncompliance with Dietary Salt Restriction and Outcomes in Chronic Heart Failure: A Propensity Score Matching Analysis from TREAT-HF Registry
}

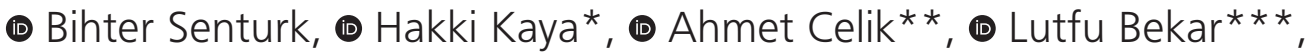

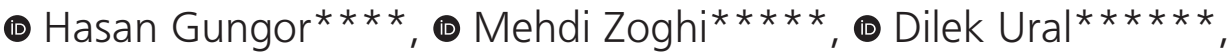 \\ (1) Yuksel Cavusoglu*******, (1) Ahmet Temizhan********, ๑ Mehmet Birhan Yilmaz \\ Dokuz Eylul University Faculty of Medicine, Department of Cardiology, Izmir, Turkey \\ ${ }^{*}$ Canakkale University Faculty of Medicine, Department of Cardiology, Canakkale, Turkey \\ **Mersin University Faculty of Medicine, Department of Cardiology, Mersin, Turkey \\ ***Hitit University Faculty of Medicine, Department of Cardiology, Çorum, Turkey \\ $* * * *$ Adnan Menderes University Faculty of Medicine, Department of Cardiology, Aydin, Turkey \\ $* * * * *$ Ege University Faculty of Medicine, Department of Cardiology, Izmir, Turkey \\ $* * * * * *$ Kocaeli University Faculty of Medicine, Department of Cardiology, Kocaeli, Turkey \\ $* * * * * * *$ Eskisehir Osmangazi University Faculty of Medicine, Department of Cardiology, Eskisehir, Turkey \\ $* * * * * * *$ Ankara City Hospital, Clinic of Cardiology, Ankara, Turkey
}

\section{Abstract}

Aim: To compare chronic heart failure patients with reduced ejection fraction (CHFrEF) who stated to comply with salt restriction in their diets versus those who did not.

Methods: Patients without salt restriction were compared to those with salt restriction regarding HF-related hospitalization (HFrH) and all-cause mortality (ACM) before and after propensity score (PS) matching analysis.

Results: The study included a total of 723 patients. 136 of them stated not to comply with salt restriction, 587 of them stated to comply with salt restriction. More frequent $\mathrm{HFrH}$ were observed in patients without salt restriction compared to those with salt restriction (75\% vs. $62.9 \%, p=0.007)$, though, ACM was similar in both groups $(29.4 \%$ vs $27.6 \%, p=0.672)$. After PS matching, HFrH during follow-up remained more frequent in those without salt restriction compared to those with salt restriction (73.7\% vs $59.3 \%$, $\mathrm{p}=0.019$ ) but ACM was not different in both groups (30.5\% vs $29.7 \%, p=0.887$ ). Noncompliance to dietary salt restriction was found as one of the independent predictors of $\mathrm{HFrH}$.

Conclusion: In CHFrEF outpatients, noncompliance to dietary salt restriction does not seem to increase the risk for ACM but it poses an increased risk for $\mathrm{HFrH}$.

Keywords: Heart failure, reduced ejection fraction, salt restriction, all-cause mortality, hospitalization

\section{Introduction}

Heart failure (HF) as a major health problem of the 21st century manifests itself among several disciplines of medicine through its multiorgan interaction. In addition, dietary salt restriction, as a non-pharmacological intervention has been recommended in the guidelines albeit with paucity of strong data until recently $(1,2)$. In the last years, the stronger recommendations of the past have been heavily argued against in the literature (3-5).

Dietary salt restriction with different thresholds is regarded as an inevitable and complementary part of the prevention of hypertension and cardiovascular disease

Yazışma Adresi/Address for Correspondence: Bihter Senturk, Dokuz Eylul University Faculty of

Medicine, Department of Cardiology, Izmir, Turkey

E-mail: drbihter@hotmail.com ORCID: orcid.org/0000-0003-3568-4476

Received: 22.01.2021 Accepted: 02.03.2021

${ }^{\circ}$ Copyright 2021 by The Medical Bulletin of istanbul Haseki Training and Research Hospital The Medical Bulletin of Haseki published by Galenos Yayınevi. 
and hence recommended in such guidelines $(6,7)$. In these guidelines, evidence is relatively well established. However, in the literature, studies evaluating the role of salt restriction in HF are small and vague, though salt restriction is recommended in HF guidelines.

Turkish Research Team in HF (TREAT-HF) is a network which undertakes multicentric observational studies in $\mathrm{HF}$ with reduced ejection fraction (HFrEF) among HF centers in Turkey (https://www.treat-hf.com/) (8-10). In our study, we aimed to compare chronic HF patients with HFrEF who stated that they complied with salt restriction in their diets versus those who did not do so.

\section{Methods}

\section{Study Design}

This study was approved by the Clinical Research Ethics Committee (date: 30.11.2010, number: 2010-01/13). In the TREAT-HF registry, consecutive prospective cohorts for the years 2013, 2014, 2015 (TREAT-HF 1, 2, 3 cohorts) recruiting chronic HFrEF outpatients were addressed. In these cohorts, chronic HFrEF outpatients were administered a questionnaire including demographics, lifestyles, attitudes by an expert after index outpatient visit. One specific question was "Do you undertake salt restriction in your diet?" with no specific threshold along with the response options of "no" and "yes". This is a subgroup analysis from TREAT-HF cohorts with a specific focus on dietary salt restriction.

HFrEF was defined as left ventricular ejection fraction (LVEF) $<40 \%$ along with HF symptoms as per guidelines recommendations $(1,2)$. Echocardiograms and laboratory data recorded within the last 3 months of index outpatient visit (if there is more than one, the closest one is considered) were considered. Chronic HFrEF was defined as LVEF $<40 \%$ in conjunction with stable HF symptoms for over one month, lack of hospitalization in previous three months, a stable dose of diuretics for over one month, optimally titrated and stable (minimum one month) doses of guideline-directed medical therapy (GDMT) involving angiotensin-converting enzyme inhibitor\&angiotensin receptor blockers, beta-blockers, mineralocorticoid receptor antagonists and ivabradine if necessary.

\section{Patient Assessment}

All patients were at ACC/AHA Stage $C$ defined as the presence of present or previous symptoms of $\mathrm{HF}$ along with structural heart disease. Functional capacities of patients were determined according to New York Heart Association (NYHA) class at time of enrolment regardless of their former status (1). All patients were under chronic outpatient follow-up by HF centers and patients needing parenteral therapy or increase in oral diuretic and/or nitrate therapy while referring to outpatient clinic were not taken into account. HF-related hospitalization ( $\mathrm{HFrH})$ during follow-up was recorded when an admitted patient needed parenteral therapy for HF symptoms and signs coupled with a minimum one-day hospitalization, recorded as "Acutely Decompensated Heart Failure" as a primary diagnosis. Patients were monitored for all-cause mortality (ACM) and $\mathrm{HFrH}$ and both events were collected every year, and all events were recorded by local investigators. Herein, HFrEF outpatients who responded to the "Do you comply with salt restriction in your diet?" question with "no" (i.e, noncompliance) were compared with "yes" for outcomes including ACM and HFrH during follow-up. A flow chart was provided for the cohort (Figure 1). Since SPSS based propensity score (PS) matching requires the presence of completely filled data set in the database, the whole cohort was narrowed to 723 patients with complete data. set mainly due to the absence of natriuretic peptides (NPs) at index admission (please refer to flow chart), as, NPs are not necessary for the diagnosis of HFrEF according to guidelines $(1,2)$ and are not routinely utilized during follow-up of stable HFrEF outpatients even among expert $\mathrm{HF}$ centers at the time of registry.

\section{Statistical Analysis}

All statistical procedures were conducted via SPSS software (version 25.0, SPSS Inc., Chicago, IL, institutional software). Continuous variables were expressed as mean \pm SD or median $\left(25^{\text {th }}-75^{\text {th }}\right.$ percentile) if there is abnormal distribution, and categorical variables as percentages. Comparisons between groups were performed, utilizing the chi-square test for categorical variables, an independent samples t-test for normally distributed continuous variables, and the Mann-Whitney $U$ test when distribution was skewed. A multivariate logistic regression analysis was performed in order to determine the independent predictors for $\mathrm{HFrH}$. The variables with $p<0.1$ obtained from univariate analysis were entered in the multivariable logistic regression model. PS matching extension was provided on top of institutional SPSS 25.0 [Thoemmes, F. (2012). Propensity score matching in SPSS. arXiv:1201.6385 (stat.AP)]. Propensity-based matching was utilized to form paired samples of patients with similar propensity scores and stratified by "yes" or "no" groups according to compliance to salt restriction. The nearest neighbor matching algorithm was used, and covariate adjustment was made for age, coronary artery disease (CAD), diabetes mellitus (DM), NTproBNP, urban life, atrial fibrillation (AF), right ventricular (RV) dilatation, ivabradine use, left atrium diameter according to data provided from the unmatched cohort. A p-value of $\leq 0.05$ was considered statistically significant. 


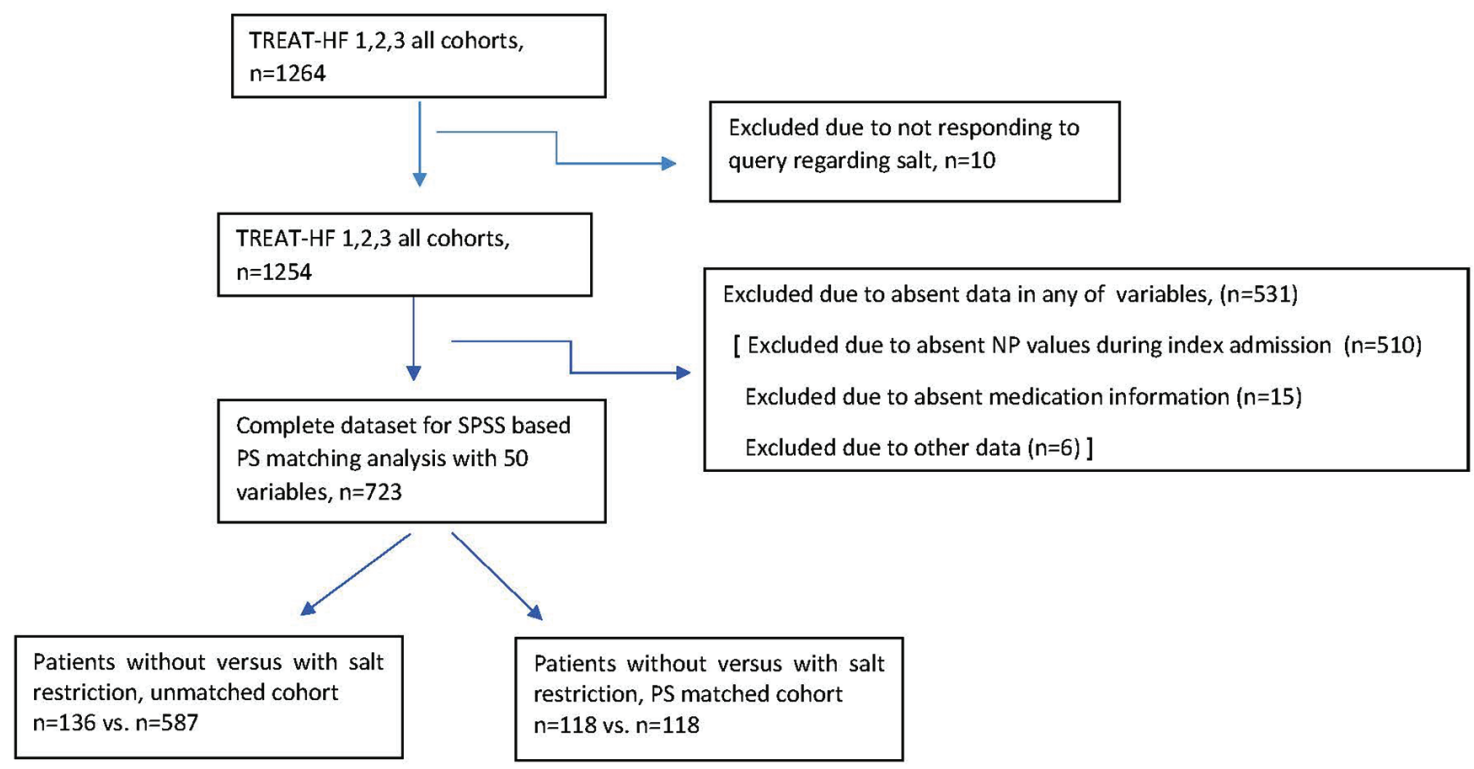

Figure 1: Flow chart of the study

TREAT-HF: Turkish Research Team heart failure, PS: propensity score, NP: Natriuretic peptide

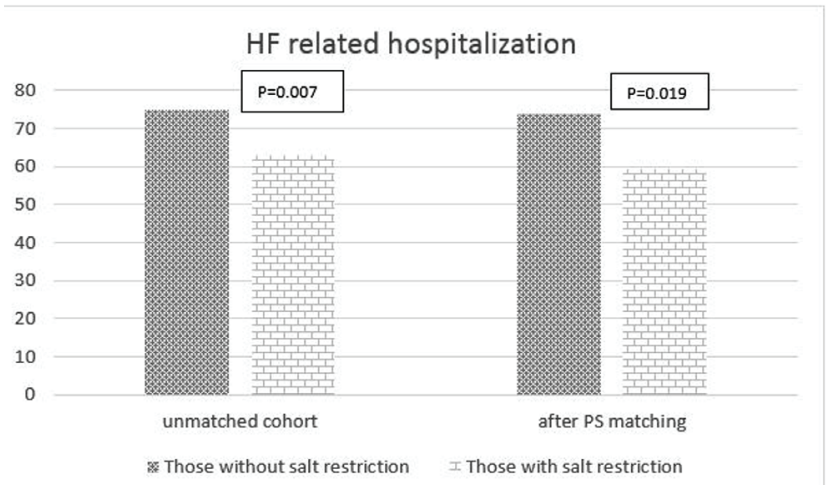

Figure 2: Frequency of HF-related hospitalization in unmatched and matched cohort

HF: Heart failure, PS: propensity score

\section{Results}

Mean follow-up of $20.2 \pm 11.8$ months up to 48 months. Upon creation of unmatched "complete dataset available cohort" for PS matching analysis on SPSS 25.0, there remained 723 chronic HF patients with reduced ejection fraction (CHFrEF) and 136 of them responded with "no" to the question of "Do you comply with salt restriction in your diet?" (i.e, those without salt restriction) and 587 of them responded with "yes" (i.e, those with salt restriction). Onethird of the cohort was females in two groups. DM and CAD were less frequent, echocardiographic RV dilatation was more frequent and NTproBNP levels were higher in patients without salt restriction along with more frequent $\mathrm{HFrH}$ compared to those with salt restriction (75\% vs. $62.9 \%, p=0.007$, Figure 2) as shown on the left panel of Table 1. Of note, ACM on follow-up was similar in both groups in the unmatched cohort $(29.4 \%$ vs. $27.6 \%$, patients without and with salt restriction respectively, $\mathrm{p}=0.672$ ). After PS matching, HFrH during follow-up remained more frequent in those without salt restriction compared to those with salt restriction (73.7\% vs. $59.3 \%$, $\mathrm{p}=0.019$, Figure 2) and ACM was not statistically different in both groups $(30.5 \%$ vs. $29.7 \%$, patients without and with salt restriction respectively, $p=0.887$ ) (right panel of Table 1).

Patients in the unmatched cohort were also classified into two as those with and without HFrH (Table 2). Upon univariate analysis, multivariable logistic regression analysis was made to obtain independent predictors of $\mathrm{HFrH}$ in the unmatched cohort (Table 3). Of note, noncompliance to dietary salt restriction was found as one of the independent predictors of HFrH along with NTproBNP levels, creatinine levels, having NYHA Class III-IV symptoms confirming PS matching analysis.

\section{Discussion}

Heart failure is characterized by neurohormonal activation, yielding sodium and water retention, which creates the issue of "congestion" as the major pathophysiology of acute HF syndromes (11). Hence, getting rid of sodium and water in the form of decongestion remains as the principle of therapy in HF (12). In addition, the association of sodium intake and fluid overload in HF brought about non-pharmacological self-care management of chronic HF via salt and fluid restriction in order to minimize the risk of acute decompensations. However, excess salt consumption is not as frequently reported as 


\begin{tabular}{|c|c|c|c|c|c|c|}
\hline Characteristics & $\begin{array}{l}\text { Patients without } \\
\text { salt restriction, } \\
\text { no group } \\
\mathrm{n}=136\end{array}$ & $\begin{array}{l}\text { Patients with salt } \\
\text { restriction, yes } \\
\text { group } \\
\mathrm{n}=587\end{array}$ & $p$ & $\begin{array}{l}\text { Patients without salt } \\
\text { restriction after PS } \\
\text { matching } n=118\end{array}$ & $\begin{array}{l}\text { Patients with salt } \\
\text { restriction after PS } \\
\text { matching } n=118\end{array}$ & $\begin{array}{l}p \text { after } \\
\text { matching }\end{array}$ \\
\hline Age (years) & $64.6 \pm 12.2$ & $62.9 \pm 12.7$ & 0.130 & $65.19 \pm 11.12$ & $65.19 \pm 11.12$ & 1.00 \\
\hline Gender (Female) n (\%) & $43(31.6 \%)$ & 187 (31.9 \%) & 0.957 & $37(31.4 \%)$ & $40(33.9 \%)$ & 0.781 \\
\hline Urban life $n(\%)$ & $22(16.2 \%)$ & $146(24.9 \%)$ & $0.032 \chi^{2}$ & $20(16.9 \%)$ & $25(21.2 \%)$ & 0.508 \\
\hline Graduation from university n (\%) & $6(4.4 \%)$ & $42(7.2 \%)$ & 0.338 & $5(4.2 \%)$ & $9(7.6 \%)$ & 0.409 \\
\hline Hypertension n (\%) & $48(35.3 \%)$ & 205 (34.9\%) & 0.921 & $42(35.6 \%)$ & $38(32.2 \%)$ & 0.680 \\
\hline Diabetes mellitus n (\%) & $24(17.6 \%)$ & $152(25.9 \%)$ & $0.046 \chi^{2}$ & $21(17.8 \%)$ & $25(21.2 \%)$ & 0.622 \\
\hline CAD n (\%) & $48(35.3 \%)$ & $300(51.1 \%)$ & $0.001 \chi^{2}$ & $43(36.4 \%)$ & $43(36.4 \%)$ & 1.00 \\
\hline NYHA 3-4 n (\%) & $61(44.9 \%)$ & $47.5(47.5 \%)$ & 0.634 & $54(45.8 \%)$ & $63(53.4 \%)$ & 0.298 \\
\hline $\mathrm{AF} n(\%)$ & $38(27.95)$ & $130(22.1 \%)$ & 0.176 & $25(21.2 \%)$ & $25(21.2 \%)$ & 1.00 \\
\hline Heart rate (bpm) & $82.26 \pm 15.88$ & $82.28 \pm 18.7$ & 0.992 & $81.41 \pm 15.55$ & $79.82 \pm 16.42$ & 0.447 \\
\hline \multicolumn{7}{|l|}{ Laboratory parameters } \\
\hline $\mathrm{BUN}(\mathrm{mg} / \mathrm{dL})$ & $39(27-62)$ & $39(25-57.4)$ & 0.602 & $39(27.18-62.25)$ & $41(26-66.23)$ & 0.819 \\
\hline Creatinine (mg/dL) & $1.39 \pm 0.66$ & $1.35 \pm 0.77$ & 0.548 & $1.40 \pm 0.67$ & $1.42 \pm 0.77$ & 0.853 \\
\hline Sodium (mmol/L) & $137.02 \pm 7.17$ & $137.85 \pm 4.20$ & 0.666 & $138.05 \pm 4.19$ & $137.61 \pm 3.95$ & 0.417 \\
\hline Potassium (mmol/L) & $4.55 \pm 0.51$ & $4.42 \pm 0.59$ & 0.279 & $4.59 \pm 0.46$ & $4.45 \pm 0.56$ & 0.212 \\
\hline NT-proBNP (pg/mL) & $2225(836-5935)$ & $1350(562-3628)$ & $0.047^{u}$ & $2769(864-5842)$ & $1862(675-4453)$ & 0.289 \\
\hline $\mathrm{Hb}(\mathrm{g} / \mathrm{dL})$ & $12.74 \pm 2.27$ & $12.51 \pm 2.21$ & 0.279 & $12.62 \pm 2.24$ & $12.26 \pm 2.12$ & 0.205 \\
\hline Hct (\%) & $39.39 \pm 6.66$ & $38.52 \pm 6.25$ & 0.152 & $39.02 \pm 6.57$ & $37.96 \pm 6.08$ & 0.199 \\
\hline \multicolumn{7}{|l|}{ Echocardiographic parameters } \\
\hline LA diameter $(\mathrm{mm})$ & $45.81 \pm 8.29$ & $44.50 \pm 6.89$ & 0.055 & $45.62 \pm 8.38$ & $44.29 \pm 6.99$ & 0.187 \\
\hline EF (\%) & $30.84 \pm 9.21$ & $31.43 \pm 8.04$ & 0.494 & $31.26 \pm 9.16$ & $31.81 \pm 8.37$ & 0.628 \\
\hline $\operatorname{LVEDD}(\mathrm{mm})$ & $57.84 \pm 9.31$ & $57.55 \pm 8.43$ & 0.709 & $57.69 \pm 9.56$ & $57.33 \pm 8.28$ & 0.759 \\
\hline RV dilatation n (\%) & $64(47.1 \%)$ & $206(35.1 \%)$ & $0.011 \chi^{2}$ & $54(45.8 \%)$ & $47(39.8)$ & 0.430 \\
\hline SPAP $(\mathrm{mmHg})$ & $43.65 \pm 13.04$ & $42.57 \pm 13.83$ & 0.410 & $44.29 \pm 6.99$ & $43.89 \pm 13.1$ & 0.802 \\
\hline \multicolumn{7}{|l|}{ Medications } \\
\hline Beta-blocker n (\%) & $110(80.9 \%)$ & $485(82.6 \%)$ & 0.620 & $96(81.4 \%)$ & $94(79.7 \%)$ & 0.870 \\
\hline ACEI/ARB n (\%) & $99(72.8 \%)$ & $430(73.3 \%)$ & 0.915 & $87(73.7 \%)$ & $83(70.3 \%)$ & 0.664 \\
\hline MRA n (\%) & $61(44.9 \%)$ & $303(51.6 \%)$ & 0.183 & $56(47.5 \%)$ & $50(42.4 \%)$ & 0.513 \\
\hline Daily Loop diuretics n (\%) & 106 (77.9 \%) & $430(73.3 \%)$ & 0.279 & $94(79.7 \%)$ & $88(74.6 \%)$ & 0.439 \\
\hline Ivabradine n (\%) & $10(7.4 \%)$ & $75(12.8 \%)$ & 0.077 & $9(7.6 \%)$ & $13(11 \%)$ & 0.503 \\
\hline Digoxin n (\%) & $30(22.1 \%)$ & $141(24 \%)$ & 0.656 & $24(20.3 \%)$ & $36(27.1 \%)$ & 0.284 \\
\hline \multicolumn{7}{|l|}{ Outcomes } \\
\hline HF-related hospitalization n (\%) & $102(75 \%)$ & $369(62.9 \%)$ & $0.007 \chi^{2}$ & $87(73.7 \%)$ & $70(59.3 \%)$ & $0.019 \chi^{2}$ \\
\hline All-cause death n (\%) & $40(29.4 \%)$ & $162(27.6 \%)$ & 0.672 & $36(30.5 \%)$ & $35(29.7 \%)$ & 0.887 \\
\hline \multicolumn{7}{|c|}{$\begin{array}{l}\text { PS: propensity score, CAD: Coronary artery disease, NYHA: New York heart association, AF: Atrial fibrillation, BUN: Blood urea nitrogen, NT-proBNP: N-terminal pro-brain } \\
\text { natriuretic peptide, Hb: Hemoglobin, Hct: Hematocrit, LA: Left atrium, EF: Left ventricular ejection fraction, LVEDD: Left ventricular end-diastolic diameter, RV: Right } \\
\text { ventricular, SPAP: Systolic pulmonary artery pressure, ACEI/ARB: Angiotensin-converting enzyme inhibitor/Angiotensin receptor blocker, MRA: Mineralocorticoid receptor } \\
\text { antagonist } \\
\chi^{2} \text { : Chi-square test, } \mathrm{n}(\%) \text {; u: Mann-Whitney U tests, median }\left(25^{\text {th }}, 75^{\text {th }} \text { percentile) }\right.\end{array}$} \\
\hline
\end{tabular}

it was thought and nonadherence with medications was more influential in one study (13). Of note, nonadherence was noted in about $10 \%$ of patients with acute $\mathrm{HF}$, and these patients had a better prognosis (14). Thereby, risk in relation to excess salt consumption might not strongly exist in the absence of high-quality of evidence as it is hypothetically considered (5). However, there are conflicting opinions in the literature (15). Universal salt reduction has long been recommended in the guidelines (16), largely owing to its proven ability to lower blood pressure, which might be driven by genetic predilection (17) and arterial stiffness in hypertensive population 
Table 2. Comparison of cHFrEF patients with and without HF-related hospitalization during follow-up

\begin{tabular}{|l|}
\hline Characteristics \\
\hline Age (years) \\
\hline Gender (Female) $\mathrm{n}(\%)$ \\
\hline Urban life $\mathrm{n}(\%)$ \\
\hline Graduation from university $\mathrm{n}(\%)$ \\
\hline Hypertension $\mathrm{n}(\%)$ \\
\hline Diabetes mellitus $\mathrm{n}(\%)$ \\
\hline CAD $\mathrm{n}(\%)$ \\
\hline Dietary \\
\hline
\end{tabular}

\begin{tabular}{l}
$\begin{array}{l}\text { Patients without HF } \\
\text { related hospitalization } \\
\mathrm{n}=252\end{array}$ \\
\hline
\end{tabular}

Patients with HF related hospitalization

$\mathrm{n}=\mathbf{4 7 1}$

Dietary salt restriction $+/-(\% / \%)$

NYHA Class III-IV $n(\%)$

AF $n(\%)$

Heart rate (bpm)

$61.82 \pm 12.52$

$79(31.3 \%)$

$64(25.4 \%)$

$18(7.1 \%)$

$81(32.1 \%)$

$62(24.6 \%)$

$112(44.4 \%)$

$63.93 \pm 12.64$

$218 / 34(86.5 \% / 13.5 \%)$

$88(34.9 \%)$

$56(22.2 \%)$

$81.78 \pm 17.62$

$151(32.1 \%)$

$104(22.1 \%)$

$30(6.4 \%)$

$172(36.5 \%)$

$114(24.2 \%)$

$236(50.1 \%)$

Laboratory parameters

\begin{tabular}{|l|}
\hline BUN $(\mathrm{mg} / \mathrm{dL})$ \\
\hline Creatinine $(\mathrm{mg} / \mathrm{dL})$ \\
\hline Sodium $(\mathrm{mmol} / \mathrm{L})$ \\
\hline Potassium $(\mathrm{mmol} / \mathrm{L})$ \\
\hline $\mathrm{NT}$-proBNP $(\mathrm{pg} / \mathrm{mL})$ \\
\hline $\mathrm{Hb}(\mathrm{g} / \mathrm{dl})$ \\
\hline
\end{tabular}

\begin{tabular}{l}
$36(22-52)$ \\
$1.17 \pm 0.61$ \\
$138.26 \pm 4.42$ \\
$4.50 \pm 0.58$ \\
$778(342-1919)$ \\
$13.08 \pm 2.08$ \\
\hline
\end{tabular}

$369 / 102$ (78.3\%/21.7\%)

$252(53.5 \%)$

$112(23.8 \%)$

$82.55 \pm 18.51$

p

0.032

0.867

0.355

0.754

0.253

0.928

0.160

$0.007 \chi^{2}$

$<0.001 \chi^{2}$

0.712

0.589

\begin{tabular}{|l|l|}
\hline $41(27-63)$ & $\mathbf{0 . 0 0 4 ^ { u }}$ \\
\hline
\end{tabular}

\begin{tabular}{|l|l|}
\hline $1.46 \pm 0.80$ & $<0.001^{\mathbf{t}}$ \\
\hline $137.67 \pm 4.06$ & 0.070 \\
\hline $4.54 \pm 0.57$ & 0.358 \\
\hline $2094(762-4870)$ & $<0.001^{\mathbf{u}}$ \\
\hline $12.27 \pm 2.24$ & $<0.001^{\mathrm{t}}$ \\
\hline
\end{tabular}

Echocardiographic parameters

\begin{tabular}{l}
\hline LA diameter $(\mathrm{mm})$ \\
\hline EF $(\%)$ \\
\hline LVEDD $(\mathrm{mm})$ \\
\hline RV dilatation $\mathrm{n}(\%)$ \\
\hline SPAP $(\mathrm{mmHg})$ \\
\hline
\end{tabular}

\section{Medications}

Beta-blocker $n(\%)$

ACEI/ARB n (\%)

MRA $n$ (\%)

Loop diuretics $n(\%)$

Ivabradine $\mathrm{n}(\%)$

Digoxin $\mathrm{n}(\%)$

\section{Outcome}

All-cause Death $n(\%)$

\begin{tabular}{l}
$44.59 \pm 7.47$ \\
\hline $32.34 \pm 8.36$ \\
\hline $57.22 \pm 8.95$ \\
\hline $96(38.1 \%)$ \\
\hline $40.83 \pm 13.76$ \\
\hline
\end{tabular}

\begin{tabular}{|l|}
\hline $44.83 \pm 7.04$ \\
\hline $30.76 \pm 8.17$ \\
\hline $57.81 \pm 8.40$ \\
\hline $174(36.9 \%)$ \\
\hline $43.82 \pm 13.54$ \\
\hline
\end{tabular}

0.667

$\mathbf{0 . 0 1 4 ^ { \mathrm { t } }}$

0.381

0.809

$\mathbf{0 . 0 0 5 ^ { \mathrm { t } }}$

\begin{tabular}{|l|l|l|}
\hline $215(85.3 \%)$ & $380(80.7 \%)$ & 0.126 \\
\hline $190(75.4 \%)$ & $339(72 \%)$ & 0.334 \\
\hline $135(53.6 \%)$ & $229(48.6 \%)$ & 0.212 \\
\hline $176(69.8 \%)$ & $360(76.4 \%)$ & 0.061 \\
\hline $32(12.7 \%)$ & $53(11.3 \%)$ & 0.628 \\
\hline $66(26.2 \%)$ & $105(22.3 \%)$ & 0.270 \\
\hline $51(20.2 \%)$ & $151(32.1 \%)$ & $\mathbf{0 . 0 0 1} \chi^{\mathbf{2}}$ \\
\hline
\end{tabular}

CHFrEF: Chronic heart failure with reduced ejection fraction, HF: Heart failure, CAD: Coronary artery disease, NYHA: New York heart association, AF: Atrial fibrillation, BUN: Blood urea nitrogen, NT-proBNP: N-terminal pro-brain natriuretic peptide, Hb: Hemoglobin, Hct: Hematocrit, LA: Left atrium, EF: Left ventricular ejection fraction, LVEDD: Left ventricular end-diastolic diameter, RV: Right ventricular, SPAP: Systolic pulmonary artery pressure, ACEI/ARB: Angiotensin-converting enzyme inhibitor/Angiotensin receptor blocker, MRA: Mineralocorticoid receptor antagonist

t: Independent sample t-test, mean \pm SD: standard deviation; $\chi^{2}$ : Chi-square test, $\mathrm{n}(\%)$; ${ }^{\mathrm{u}}$ : Mann-Whitney $\mathrm{U}$ tests, median $\left(25^{\text {th }}, 75^{\text {th }}\right.$ percentile), SD: Standard deviation

(18). Nevertheless, it was recently shown in the PURE study that moderation of salt intake between $3 \mathrm{~g} /$ day- 6 $\mathrm{g} /$ day was associated with a lower risk of mortality and cardiovascular events compared to either higher or lower levels of salt intake (19). Hence, strict restriction of salt intake does not seem to work in the right direction as expected in overall population. However, HF guidelines adopt salt restriction to a larger extent and recommend salt restriction in the absence of strong data (20). More interestingly, 15 years ago, Alvelos et al. (21) reported in an elegantly designed study that low sodium diet in chronic HFrEF resulted in "activation of anti-natriuretic the antinatriuretic and antidiuretic systems in HF patients". Five years later, Parrinello et al. (22) reported that moderation of sodium intake among HF patients after decompensation was associated with better outcomes compared to low 


\begin{tabular}{|c|c|c|c|}
\hline & $p$ & OR & $95 \% \mathrm{Cl}$ \\
\hline NTproBNP (pg/mL) & $<0.001$ & 1.001 & $1.000-1.001$ \\
\hline $\begin{array}{l}\text { Noncompliance with dietary salt } \\
\text { restriction }\end{array}$ & 0.046 & 1.597 & $1.009-2.527$ \\
\hline Creatinine (mg/dL) & 0.004 & 1.701 & $1.189-2.434$ \\
\hline NYHA Class III-IV & 0.006 & 1.631 & $1.150-2.313$ \\
\hline
\end{tabular}

Variables entered in the logistic regression model: Age, NTproBNP, dietary salt restriction, NYHA Class III-IV, BUN, creatinine, Hemoglobin, left ventricular ejection fraction, systolic pulmonary artery pressure, sodium, daily loop diuretic use. NT-proBNP: N-terminal pro-brain natriuretic peptide, HF: Heart failure, NYHA: New York heart association, BUN: Blood urea nitrogen, Cl: confidence interval

sodium, which yielded activation of neurohormones and cytokines (23). Later on, Aliti et al. (24) reported that aggressive salt restriction did not enhance weight loss or clinical stability in initial days and concluded that salt restriction in patients with HF was "unnecessary" though, individualized restriction of salt consumption to $5 \mathrm{~g} /$ day (moderation) along with fluid restriction might improve signs and symptoms of HF (25). In a more recent paper serially evaluating salt intake of chronic HF patients with food frequency questionnaire, patients were arbitrarily classified into two groups as restricted salt ( $<2.5 \mathrm{~g} /$ day) and unrestricted salt ( $>2.5 \mathrm{~g} /$ day) and it was found that restricted salt intake was associated with poorer outcomes (26). On the contrary, Arcand et al. (27) reported in chronic HF patients that highest tertile of salt consumption (mean $3.8 \mathrm{~g}$ sodium/day) was associated with 3.5 times increased risk for mortality during 3-year follow-up. Therefore, in the presence of different thresholds in different studies, it remains to be established how much salt should be regarded as "too much" given that weather, sweating, background salt sensitivity etc. all seem to influence any significant interaction in the body (28). It is interesting to note in one palatability study that chronic HF patients preferred food with a higher concentration of salt more frequently compared to healthy controls (29). This issue was defined as a "hedonic shift" in relation to impaired recognition of salt taste, particularly after acute HF (30). Furthermore, after discharge from the hospital, the educated behavioral pattern of HF patients fora low sodium diet has recently been shown to decrease significantly in 3 months (31). Hence, it may be reasonable to individualize non-pharmacological management, particularly salt intake and to avoid strict numeric thresholds since the majority of these patients have altered sensation and does not follow salt restriction soon after hospitalization, and then, the best strategy might be to let the patient decide according to his/her palatability. Of note, Sodium-HF trial is expected to provide scientific evidence to strict salt restriction in patients with chronic HF, as it evaluates the efficacy of strict dietary sodium reduction ( $<1.5 \mathrm{~g} /$ day) in comparison to usual care for patients with chronic HF (32).

In the current analysis enrolling chronic HFrEF patients from expert HF centers in Turkey, we found an increased risk for $\mathrm{HFrH}$ in those without salt restriction, along with no increased risk for ACM, though, "no" group, i.e., those without salt restriction were, less frequently in urban life, higher N-terminal pro-brain natriuretic peptide, more frequent RV dilatation on echocardiogram in the whole cohort. Upon creation of an unmatched cohort with the complete dataset, similar results were obtained. Then, PS matching analysis in SPSS along with adjustment for covariates resulted in more $\mathrm{HFrH}$ in those without salt restriction along with no difference in ACM on followup, which is discrepant with the recent data Doukky $R$ and coworkers (26) though not fully supporting the data Arcand and coworkers (27), since we did not observe any negative or positive signal with regard to ACM, However, $\mathrm{HFrH}$ was influenced by salt restriction in both analyses. If our study had assessed cardiac deaths in addition to ACM, perhaps cardiac deaths would be found to be more common in patients without salt limitation. We found $\mathrm{ACM}$ was more frequent in patients with $\mathrm{HFrH}$. We think this significant difference may be due to cardiac deaths.

\section{Study Limitations}

Several confounders might have been missed in this study. First of all, "no" response might not mean consistent "no" or vice versa during follow-up. Hence, many patients might have crossed to "no" group from "yes" group according to patients' congestion status as it is not easy to keep up with salt restriction continuously, and hence, any potential benefit with regard to ACM might have been missed. Furthermore, only ACM was assessed; cardiac deaths were not separately assessed in our study. In the cohort, the "no" group was smaller. Since the centers participating in the TREAT-HF cohorts were expert HF centers in Turkey, there was a bias for stronger motivation for non-pharmacological interventions compared to overall physician attitude. However, initial enthusiasm for salt restriction decreased in the literature during the enrolment phase of the registry, hence changing environment might have diluted the effect of the findings. Besides, there might be other reasons for not complying with salt restriction such as previous hyponatremia. GDMT use was evaluated only at baseline, however, any improvement in GDMT during follow-up and better adherence to GDMT could potentially alter prognosis. Since the patient cohort was made up of relatively stable and chronic outpatients with HFrEF and without recent decompensation, there might have been a selection bias for excluding HFrEF 
patients who might benefit from salt restriction. Hence, the potential role of the salt restriction in patients with more progressive disease, more rapid course or more fluctuating course remains still remains to be established. Besides, the NYHA Class IV group was small and was mainly made up of ambulatory patients since salt restriction might have some value in these patients. Diagnosis of HFrH was not adjudicated independently and some events outside the participant hospital might have been underestimated since some HF-related events might have been missed, at least as the primary diagnosis, in other hospitals. Last but not least, relying on a qualitative measure rather than measuring urine sodium or a specific food questionnaire could be a major limitation. However, urine sodium excretion is determined by individual status with large inter-individual variations and is questionable in the setting of chronic diuretic use; hence it is not a simple biomarker (33).

\section{Conclusion}

"Declaring" noncompliance with dietary salt restriction was associated with increased HFrH risk in chronic HFrEF outpatient population, though, ACM remained unaffected.

\section{Authorship Contributions}

Concept: B.S., H.K., A.C., L.B., H.G., M.Z., D.U., Y.C., A.T., M.B.Y., Design: B.S., L.B., H.G., D.U., Y.C., A.T., M.B.Y., Data Collection or Processing: B.S., H.K., A.C., L.B., H.G., M.Z., D.U., Y.C., A.T., M.B.Y., Analysis or Interpretation: B.S., L.B., H.G., D.U., Y.C., A.T., M.B.Y., Literature Search: B.S., H.K., A.C., L.B., H.G., M.Z., D.U., Y.C., A.T., M.B.Y., Writing: B.S., M.B.Y.

Conflict of Interest: No conflict of interest was declared by the authors.

Financial Disclosure: The authors declared that this study received no financial support.

\section{References}

1. Yancy CW, Jessup M, Bozkurt B, et al. 2013 ACCF/AHA guideline for the management of heart failure: a report of the American College of Cardiology Foundation/American Heart Association Task Force on Practice Guidelines. J Am Coll Cardiol 2013;62:147-239.

2. Ponikowski P, Voors AA, Anker SD, et al. 2016 ESC Guidelines for the diagnosis and treatment of acute and chronic heart failure: The Task Force for the diagnosis and treatment of acute and chronic heart failure of the European Society of Cardiology (ESC). Developed with the special contribution of the Heart Failure Association (HFA) of the ESC. Eur J Heart 2016;37:2129-200.

3. DiNicolantonio JJ, Chatterjee S, O'Keefe JH. Dietary Salt Restriction in Heart Failure: Where Is the Evidence? Prog Cardiovasc Dis 2016;58:401-6.

4. Hummel SL, Konerman MC. Dietary Sodium Restriction in Heart Failure: A Recommendation Worth its Salt? JACC Heart Fail 2016;4:36-8.
5. Mahtani KR, Heneghan C, Onakpoya I, et al. Reduced Salt Intake for Heart Failure: A Systemat Review. JAMA Intern Med 2018;178:1693-700.

6. Williams B, Mancia G, Spiering W, et al. 2018 ESC/ESH Guidelines for the management of arterial hypertension. Eur Heart J 2018;39:3021-104.

7. Piepoli MF, Hoes AW, Agewall S, et al. 2016 European Guidelines on cardiovascular disease prevention in clinical practice: The Sixth Joint Task Force of the European Society of Cardiology and Other Societies on Cardiovascular Disease Prevention in Clinical Practice (constituted by representatives of 10 societies and by invited experts)Developed with the special contribution of the European Association for Cardiovascular Prevention \& Rehabilitation (EACPR). Eur Heart J 2016;37:2315-81.

8. Kaya H, Beton O, Acar G, et al. Influence of influenza vaccination on recurrent hospitalization in patients with heart failure. Herz 2017;42:307-15.

9. Kaya H, Refiker Ege M, Zorlu A, et al. Relationship between functional capacity and socioeconomic status in a cohort of Turkish heart failure patients. Turk Kardiyol Dern Ars 2016;44:481-7.

10. Cavusoglu $\mathrm{Y}$, Kaya H, Eraslan S, Yilmaz MB. Hyponatremia is associated with occurrence of atrial fibrillation in outpatients with heart failure and reduced ejection fraction. Hellenic J Cardiol 2019;60:117-21.

11. Gheorghiade $M$, Follath $F$, Ponikowski $P$, et al. Assessing and grading congestion in acute heart failure: a scientific statement from the acute heart failure committee of the heart failure association of the European Society of Cardiology and endorsed by the European Society of Intensive Care Medicine. Eur J Heart Fail 2010;12:423-33.

12. Mullens W, Damman K, Harjola VP, et al. The use of diuretics in heart failure with congestion - a position statement from the Heart Failure Association of the European Society of Cardiology. Eur J Heart Fail 2019;21:137-55.

13. Borke JA, Wyer PC. Eating a larger number of high-salt foods is not associated with short-term risk of acute decompensation in patients with chronic heart failure. J Emerg Med 2013;44:36-45.

14. Ambardekar AV, Fonarow GC, Hernandez $A F$, et al. Characteristics and in-hospital outcomes for nonadherent patients with heart failure: findings from Get With The Guidelines-Heart Failure (GWTG-HF). Am Heart J 2009;158:644-52.

15. Messerli FH, Suter T, Anwander MT. Postholiday Heart Failure Triggered by Dietary Salt Excess. JAMA Intern Med 2019;179:1006-7.

16. National Clinical Guideline Centre (UK). Hypertension: The Clinical Management of Primary Hypertension in Adults: Update of Clinical Guidelines 18 and 34 [Internet]. London: Royal College of Physicians (UK); 2011 Aug. 
17. Imaizumi $T$, Ando $M$, Nakatochi $M$, et al. Association of interactions between dietary salt consumption and hypertension-susceptibility genetic polymorphisms with blood pressure among Japanese male workers. Clin Exp Nephrol 2017;21:457-64.

18. D'Elia L, Galletti F, La Fata E, Sabino P, Strazzullo P. Effect of dietary sodium restriction on arterial stiffness: systematic review and meta-analysis of the randomized controlled trials. J Hypertens 2018;36:734-43.

19. O'Donnell M, Mente A, Rangarajan S, et al. Urinary sodium and potassium excretion, mortality, and cardiovascular events. N Engl J Med 2013;371:612-23.

20. Hunt SA. ACC/AHA 2005 guideline update for the diagnosis and management of chronic heart failure in the adult: a report of the American College of Cardiology/American Heart Association Task Force on Practice Guidelines (Writing Committee to Update the 2001 Guidelines for the Evaluation and Management of Heart Failure). J Am Coll Cardiol 2005;46:1-82.

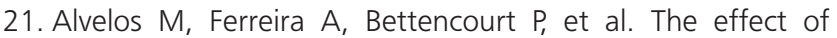
dietary sodium restriction on neurohumoral activity and renal dopaminergic response in patients with heart failure. Eur J Heart Fail 2004;6:593-9.

22. Parrinello G, Di Pasquale P, Licata G, et al. Long-term effects of dietary sodium intake on cytokines and neurohormonal activation in patients with recently compensated congestive heart failure. J Card Fail 2009;15:864-73.

23. Paterna S, Parrinello G, Cannizzaro S, et al. Medium term effects of different dosage of diuretic, sodium, and fluid administration on neurohormonal and clinical outcome in patients with recently compensated heart failure. Am J Cardiol 2009;103:93-102.

24. Aliti GB, Rabelo ER, Clausell N, Rohde LE, Biolo A, Beckda-Silva L. Aggressive fluid and sodium restriction in acute decompensated heart failure: a randomized clinical trial. JAMA Intern Med 2013;173:1058-64.

25. Philipson H, Ekman I, Forslund HB, Swedberg K, Schaufelberger M. Salt and fluid restriction is effective in patients with chronic heart failure. Eur J Heart Fail 2013;15:1304-10.

26. Doukky R, Avery E, Mangla A, et al. Impact of Dietary Sodium Restriction on Heart Failure Outcomes. JACC Heart Fail 2016;4:24-35.

27. Arcand J, Ivanov J, Sasson A, et al. A high-sodium diet is associated with acute decompensated heart failure in ambulatory heart failure patients: a prospective follow-up study. Am J Clin Nutr 2011;93:332-7.

28. Messerli FH, Neagoe A, Walpoth BN. How much salt is too much salt? Anatol J Cardiol 2019;22:2-4.

29. de Souza JT, Matsubara LS, Menani JV, Matsubara BB, Johnson AK, De Gobbi Jl. Higher salt preference in heart failure patients. Appetite 2012;58:418-23.

30. Cohen LP, Hummel SL, Maurer MS, López-Pintado S, Wessler JD. Salt Taste Recognition in a Heart Failure Cohort. J Card Fail 2017;23:538-44.

31. Riegel B, Lee S, Hill J, et al. Patterns of adherence to diuretics, dietary sodium and fluid intake recommendations in adults with heart failure. Heart Lung 2019;48:179-85.

32. Colin-Ramirez E, Ezekowitz JA. SODIUM-HF investigators. Rationale and design of the Study of Dietary Intervention Under $100 \mathrm{MMOL}$ in Heart Failure (SODIUM-HF). Am Heart J 2018;205:87-96.

33. Martens P, Dupont M, Verbrugge FH, et al. Urinary Sodium Profiling in Chronic Heart Failure to Detect Development of Acute Decompensated Heart Failure. JACC Heart Fail 2019;7:404-14. 